\title{
Four Patients Undergoing Labioplasty and Lip Reconstruction for Lip Deformity
}

\section{Kazuhide Matsunaga1*, Akifumi Enomoto1, Takeshi Shimoide1, Sanae Iwasaki', Takao Mukai1, Noritaka Isogai ${ }^{2}$, Suguru Hamada ${ }^{1}$}

\author{
${ }^{1}$ Department of Oral and Maxillofacial Surgery, Faculty of Medicine, Kindai University, Osaka, Japan \\ ${ }^{2}$ Department of Plastic and Reconstructive Surgery, Faculty of Medicine, Kindai University, Osaka, Japan \\ Email: *kamatsunaga@med.kindai.ac.jp
}

How to cite this paper: Matsunaga, K., Enomoto, A., Shimoide, T., Iwasaki, S., Mukai, T., Isogai, N. and Hamada, S. (2018) Four Patients Undergoing Labioplasty and Lip Reconstruction for Lip Deformity. Surgical Science, 9, 97-108. https://doi.org/10.4236/ss.2018.93010

Received: January 13, 2018

Accepted: March 10, 2018

Published: March 13, 2018

Copyright $\odot 2018$ by authors and Scientific Research Publishing Inc. This work is licensed under the Creative Commons Attribution International License (CC BY 4.0).

http://creativecommons.org/licenses/by/4.0/

\begin{abstract}
We managed four patients who had neoplastic, posttraumatic, atrophic and postoperative lip deformity, respectively. Case 1: The patient had a hemangioma $(17 \times 14 \mathrm{~mm})$ confined to the vermilion part of the lower lip. We performed the wedge excision of the tumor and labioplasty. The postoperative appearance and function of the lip were satisfactory. Case 2: The patient had posttraumatic deformity of the upper lip. We performed correction by Cronin's triangular flap method. The postoperative appearance of the lip was satisfactory. Case 3: The patient had severe atrophy of the upper lip and difficulty closing mouth due to progressive hemifacial atrophy. We performed reconstruction of the upper lip on the atrophic side with a cross-lip vermilion flap from the non-atrophic lower lip. The postoperative appearance and function of the lip were satisfactory. Case 4: The patient had postoperative lower lip deformity and difficulty closing mouth after tumor resection and reconstruction with a myocutaneous pectoralis major flap. We performed debulking of the bulky flap and covered the raw surface with a local rotation flap. The postoperative lip closure was satisfactory. In patients with lip deformity, it is important to perform appropriate correction or reconstruction according to each patient's clinical features in order to achieve satisfactory cosmetic and functional outcomes.
\end{abstract}

\section{Keywords}

Lip Deformity, Labioplasty, Lip Reconstruction

\section{Introduction}

The cause of lip deformity can be classified as congenital [1], atrophic [2], neop- 
lastic [3]-[8], posttraumatic [9] [10] or postoperative [11]. Lip deformity often results in cosmetic and functional impairment. Therefore, it is necessary to provide appropriate treatment for patients with lip deformity while considering cosmetic and functional outcomes. With regard to neoplastic lip deformity, many studies on lip hemangioma have been reported [3]-[8]. According to the size of the tumor, lip hemangioma can be removed by wedge resection [3], two-stage resection [4] or reconstruction after resection using various techniques [5] [6] [7] [8]. With regard to posttraumatic lip deformity, scarring and the loss of lip cause shortness of the lip [10]. It is necessary for satisfactory cosmetic outcomes to remove the scar and extend the amount of the lip. The treatment of atrophic lip deformity due to progressive hemifacial atrophy has been reported [2]. A cross-lip vermilion flap of the lower lip has been reported as a useful technique to correct the form in the presence of upper lip atrophy [2]. For postoperative lip deformity, some patients who underwent after oral cancer resection and reconstruction with flaps have been reported [11]. It is necessary for satisfactory cosmetic and functional outcomes to perform revision of the flap. Here we report our experience with four patients who have lip deformity with a neoplastic, posttraumatic, atrophic and postoperative etiology, respectively.

\section{Case Report}

\subsection{Case 1}

Patient: a 57-year-oldman.

Chief complaint: Mass lesion on the left side of the lower lip.

History: The patient noticed a small mass on the left side of the lower lip about 20 years ago. The mas gradually became larger until it causes both cosmetic and functional problems. In 2017, he consulted us for resection of the lesion and correction of his lower lip.

Lip findings at initial consultation: A mass, which did not cross the vermilio-cutaneous junction, was seen on the left side of the lower lip. It measured 17 $\times 14 \mathrm{~mm}$, and hemispherical with a dark red color (Figure 1(a), Figure 1(b)).

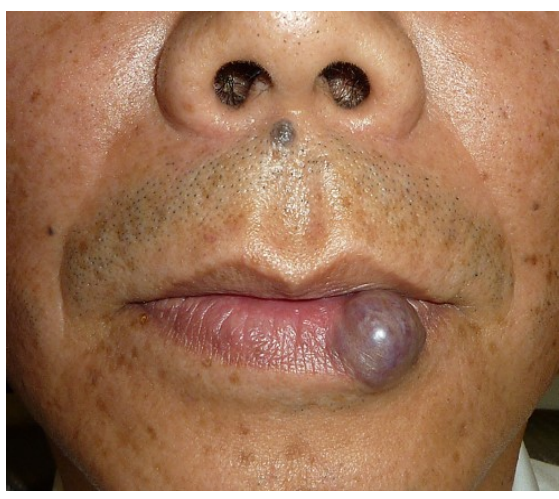

(a)

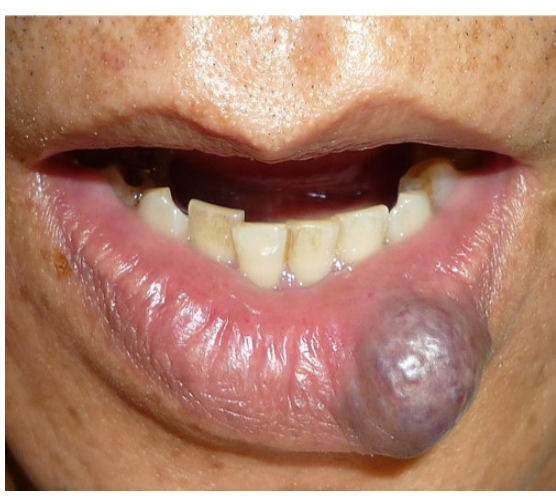

(b)

Figure 1. Lip finding at initial consultation in case 1. (a): There is a mass measuring $17 \times$ 14 on the left side of the lower lip; (b): The mass is limited to the vermilion part of the lip. 
Imaging findings at initial consultation: T2-weighted image MRI revealed a high signal intensity mass on the left side of lower lip (Figure 2(a), Figure 2(b)).

Diagnosis: Hemangioma of the lower lip

Operation: The vermilion border of lower lip was marked and a spindle-sharped incision line was made around the tumor (Figure 3(a)). A feeding vessel was ligated and the tumor was removed (Figure $3(\mathrm{~b})$ ). Then the orbicular oris muscle of the lower lip was sutured and reconstructed (Figure 3(c)). Finally, the

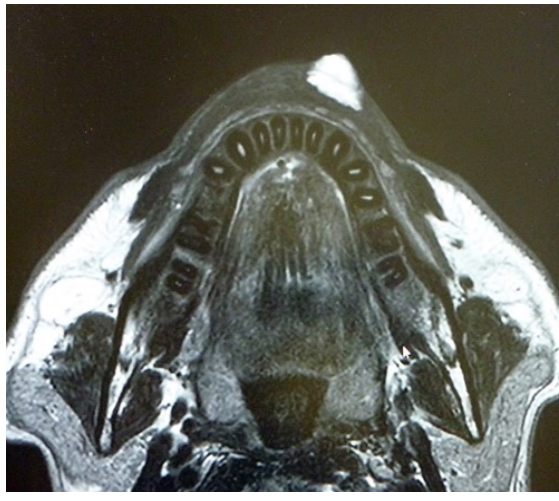

(a)

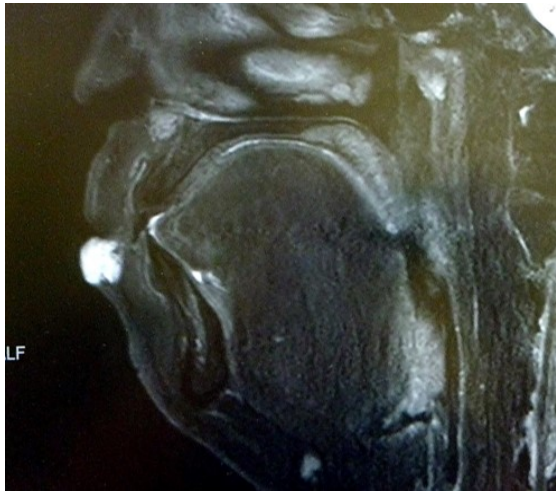

(b)

Figure 2. Imaging finding at initial consultation in case 1. T2-weighted MRI revealed a high signal intensity mass in the lower lip on the left side.

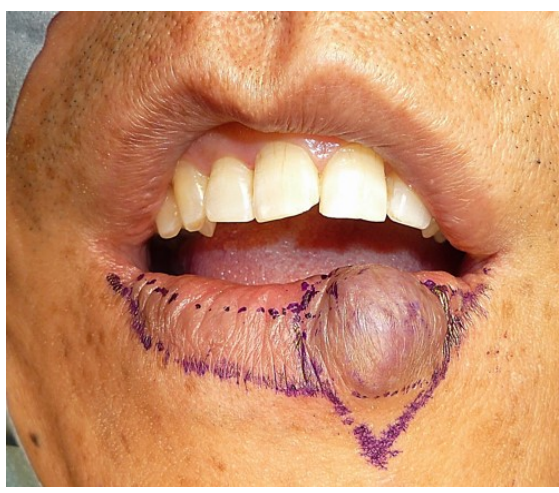

(a)

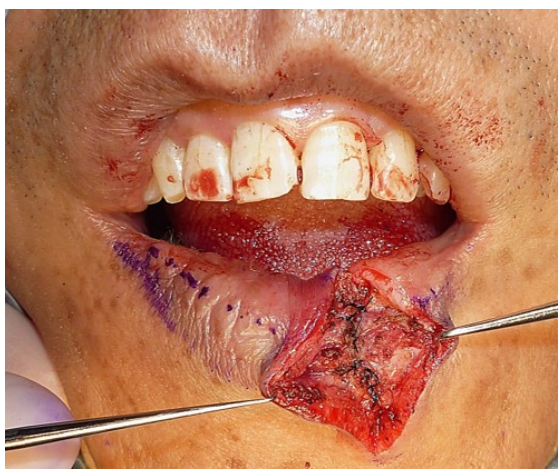

(c)

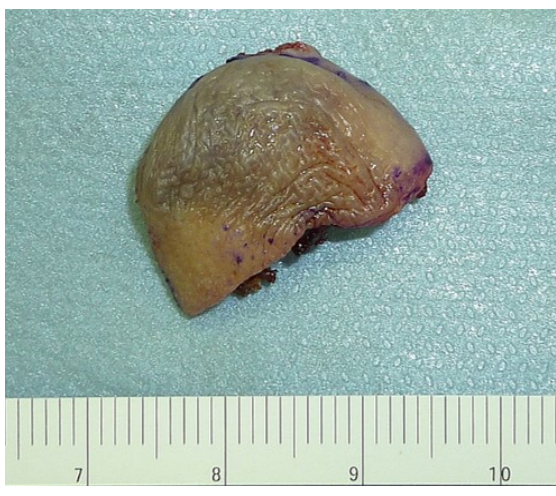

(b)

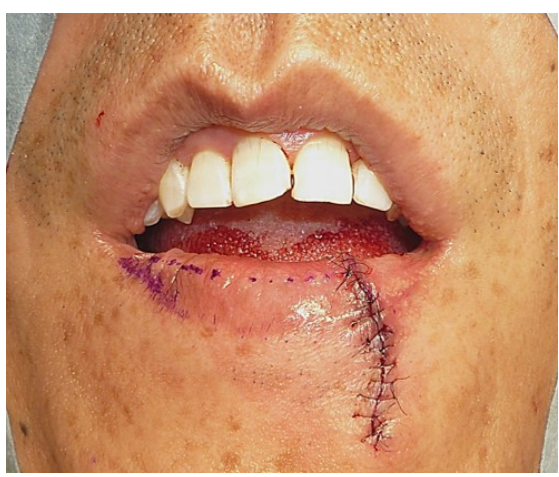

(d)

Figure 3. Operative findings in Case 1. (a): Incision line; (b): Resected lesion; (c): Suturing orbicularis oris; (d): Immediate postoperative findings. 
vermilion part of the lip, the vermilion border, and the adjacent skin were sutured to achieve symmetrical reconstruction of the lower lip (Figure 3(d)).

Postoperative course: Both the sharp and function of the lip were satisfactory at 4 months after surgery (Figure 4(a), Figure 4(b)).

\subsection{Case 2}

Patient: a 44-year-oldman.

Chief complaint: Posttraumatic upper lip deformity.

History: In 2007, he injured his upper lip by falling off a bicycle. The injury was sutured at another hospital on the same day. However, he noted deformity of the upper lip on the left side and consulted us for correction at 4 months after injury.

Lip findings at initial consultation: On the left side of the upper lip, the vermilion border demonstrated elevation toward the nasal base and there was marked contracture of the upper lip (Figure 5).

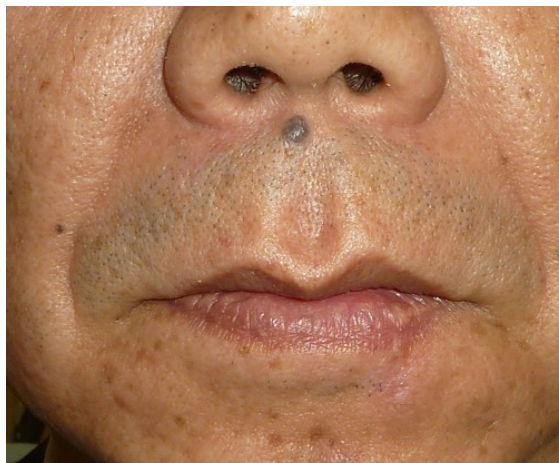

(a)

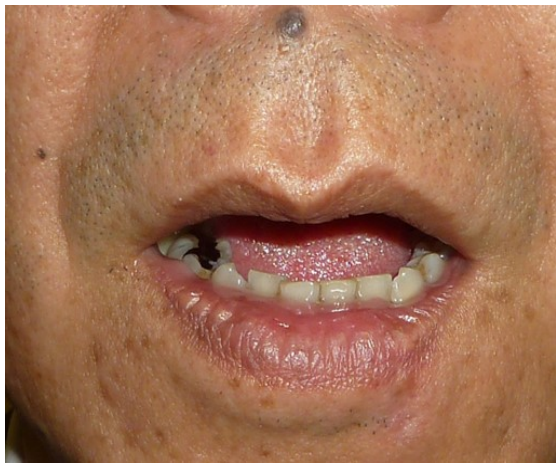

(b)

Figure 4. Postoperative findings in case 1. (a), (b): Cosmetic and functional outcomes are good at 4 months postoperatively.

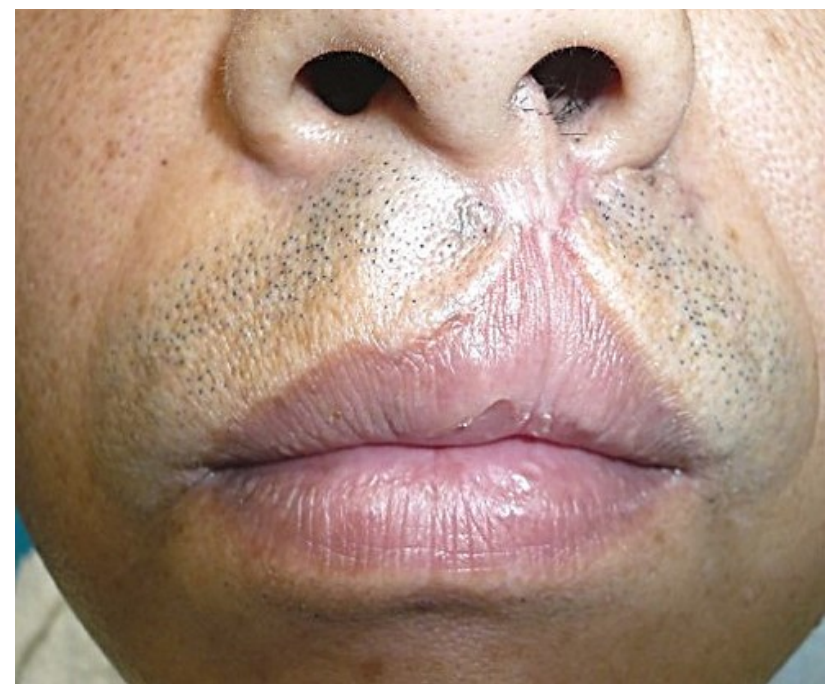

Figure 5. Lip findings at initial consultation in case 2. The left vermilion border is elevated and there is severe contracture of the upper. 
Diagnosis: Posttraumatic contracture of the upper lip on the left side

Operation: An incision was designed according to Cronin's triangular-flap technique (Figure 6(a), Figure 6(b)) [12] [13] [14]. After the skin incision was made, the orbicular or is muscle of the upper lip was sutured to achieve reconstruction in the anatomical position (Figure 6(c)). Subsequently, the white and vermilion parts of lip on the left side were sutured to restore symmetry (Figure 6(d)).

Postoperative course: At 1 year postoperatively, the cosmetic outcome was generally satisfactory, although the vermilion part of the lip was slightly bulky on the left side (Figure 7).

\subsection{Case 3}

Patient: a 22-year-old woman.

Chief complaint: Atrophy of the upper lip and difficulty closing the left side of the mouth.

History: She first noticed left-sided facial deformity at 14 years and progressive hemifacial atrophy was diagnosed at that time. Atrophy of the upper lip became severe when she was 19 years old and closing the mouth gradually became more difficult due to lip atrophy. In 2013 she consulted us for correction of her upper lip.

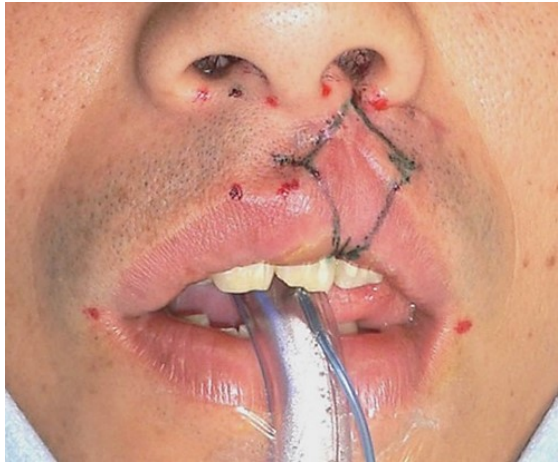

(a)

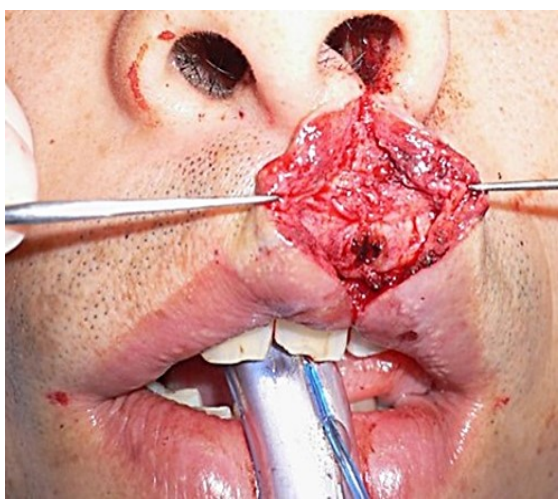

(c)

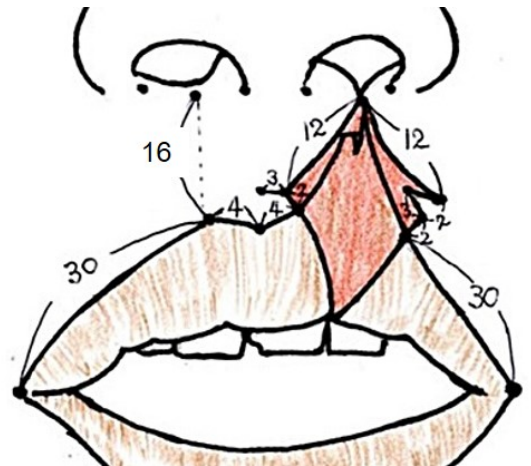

(b)

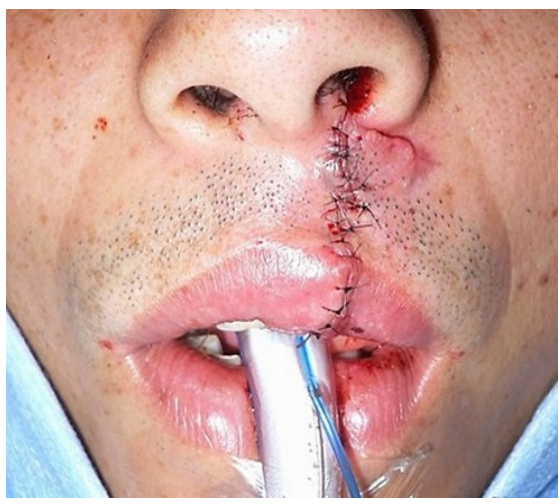

(d)

Figure 6. Operative findings in case 2. (a): Incision line; (b): Detailed design of the skin incision; (c): Suturing orbicularis oris; (d): Immediate postoperative findings. 
Lip findings at initial consultation: The center of the lips was shifted to the left, with advanced atrophy of both lips on the left side. The maxillary teeth were exposed on the left side when the mouth was relaxed (Figure 8(a)).

Preoperative imaging findings: Both the body and arch of the left zygoma showed atrophy. There was also atrophy of the body, angle, and ramus of the left mandible (Figure 8(b)).

Diagnosis: Atrophy of the lips due to progressive hemifacial atrophy.

Operation: A horizontal incision was made along the lower edge of the vermilion border of the upper lip on the atrophic side (Figure 9(a)) [15]. Severe atrophy of the orbicular oris muscle of the upper lip was noted on the left side. A longitudinal wedge flap (that tapered as it neared commissure) was developed along the vermilion border of the lower lip on the non-atrophic side (Figure 9(b)). This vermilion flap was raised with part of orbicular oris muscle, rotated $180^{\circ}$, and inserted into the transverse incision in the upper side (Figure 9(c)). Flap release was performed after 14 days (Figure 9(d)).

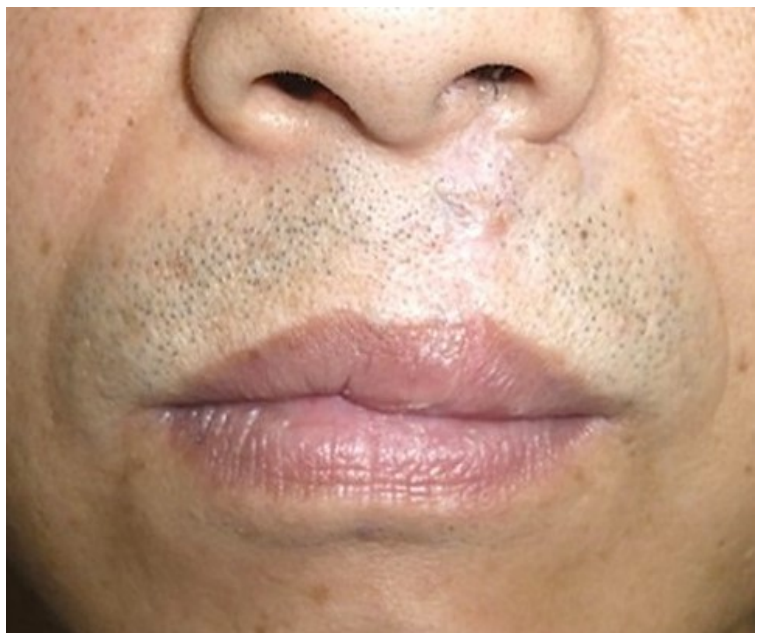

Figure 7. Postoperative finding in case 2. The appearance of the lips is generally satisfactory at 1 year postoperatively.

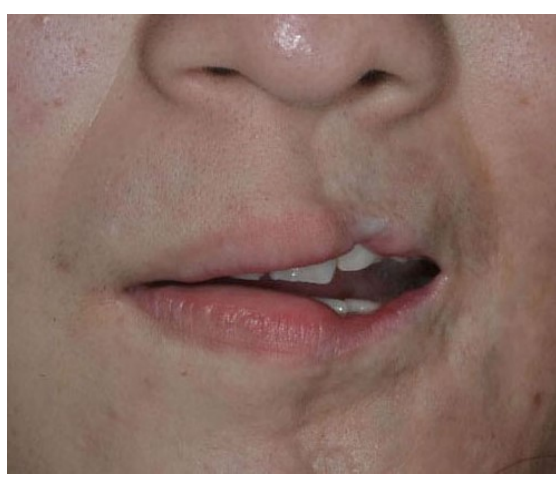

(a)

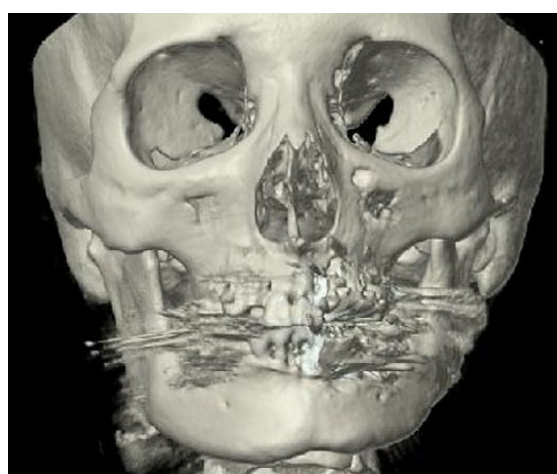

(b)

Figure 8. Lip and imaging findings at initial consultation in case 3. (a): The upper and lower lips demonstrate advanced atrophy on the left side; (b): The left zygomatic and the left mandible show atrophy. 
Postoperative course: The flap showed good viability without atrophy and deformity of the upper lip being corrected on the affected side. Both the cosmetic outcome and lip closure were satisfactory at 2 years postoperatively (Figure 10(a), Figure 10(b)).

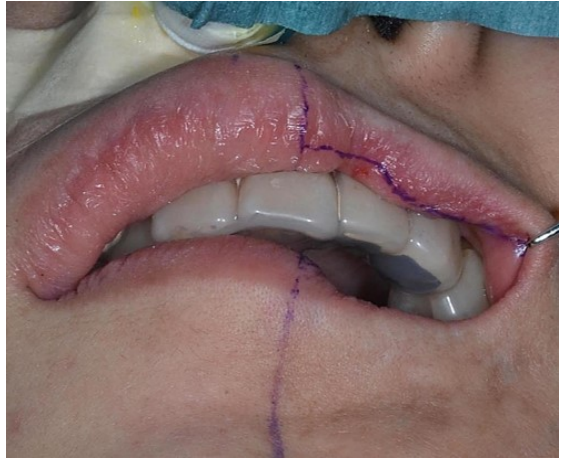

(a)

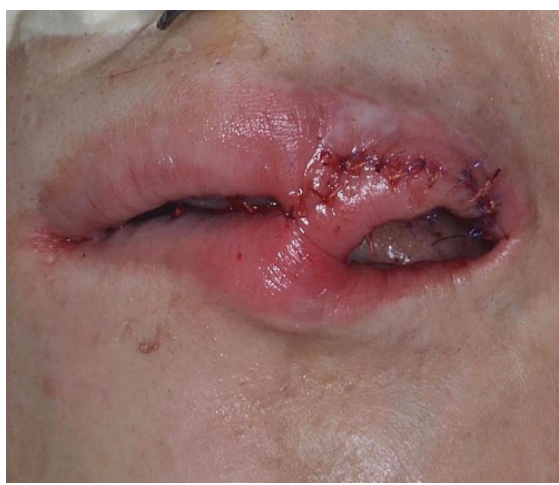

(c)

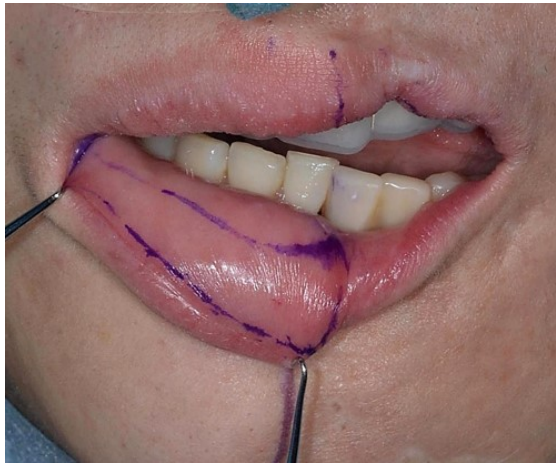

(b)

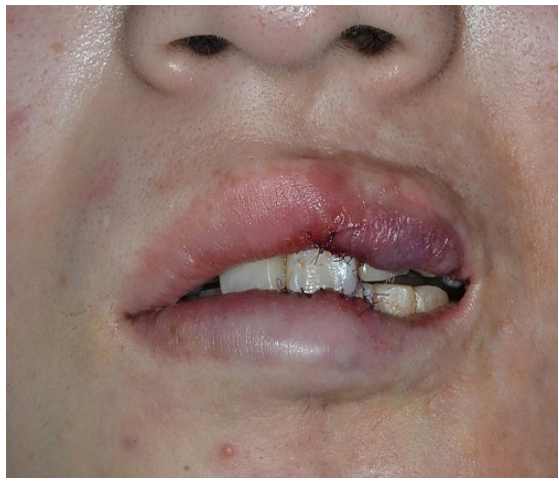

(d)

Figure 9. Operative findings in case 3. (a): A transverse incision is made along the lower edge of the vermilion border of the upper lip on the atrophic side. (b): A flap is developed along the vermilion border as a longitudinal wedge that tapers towards the commissure. (c): The vermilion flap is rotated $180^{\circ}$ and insert into the transverse incision in the upper lip. (d): The flap is released at 14 days postoperatively.

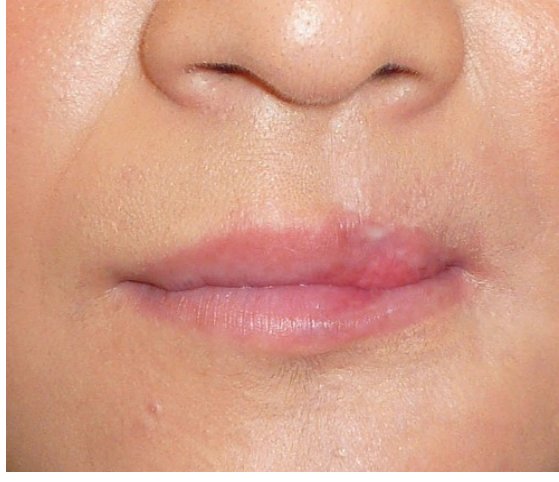

(a)

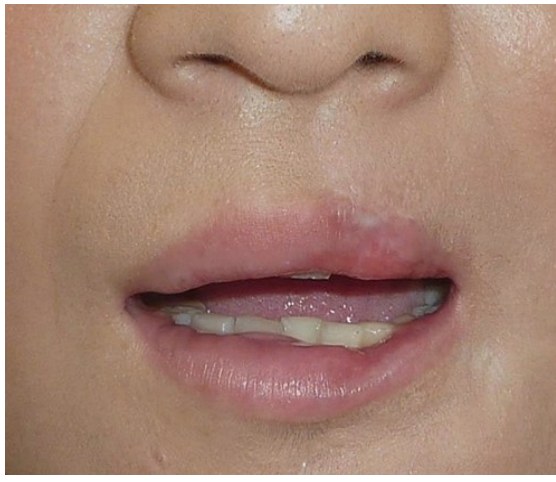

(b)

Figure 10. Postoperative findings case 3. (a): Atrophy and deformity of the upper lip are not recognizable on the affected side at 2 years postoperatively. (b): Both lip shape and lip closure are satisfactory on the affected side at 2 years postoperatively. 


\subsection{Case 4}

Patient: a 79-year-old man.

History: In 2015 the patient underwent resection of the lower lip, buccal mucosa and the mandibular gingiva on the left side to remove squamous cell carcinoma, followed by reconstruction with a myocutaneous pectoralis major flap. However, closing the mouth became increasingly difficult over time and he presented for correction of the upper lip at 5 months postoperatively.

Lip findings at initial consultation: The flap was remarkable bulky and lower lip was everted on the right. There was marked depression at the junction between lip and flap (Figure 11(a), Figure 11(b)).

Diagnosis: Postoperative contracture of lower lip.

Operation: The incision line was drawn around flap and junction between flap and lower lip (Figure 12(a)). Defatting of the flap was performed (Figure 12(b)). After the volume of the original flap was reduced, a local rotation flap was raised from the mental region (Figure 12(c)), it was used to cover raw surface created by revision surgery (Figure 12(d)).

Postoperative course: Closing the mouth was satisfactory at 2 years postoperatively, although the flap was still slightly bulky (Figure 13).

\section{Discussion}

\subsection{Lip Deformity due to Hemangioma}

A hemangioma of lip requires surgical resection to restore normal labial anatomy. Various methods have been reported for removal of hemangioma, depending on the size of the lesion. Small hamangiomas can be removed by wedge resection [3] or by two-stage resection (vertically oriented wedge resection, following by horizontal wedge along the vermilio-cutaneous junction) [4]. Medium-size lesions $(15 \sim 25 \times 18 \sim 50 \mathrm{~mm})$ require closure of the defect with a labial mucosal advancement flap after resection [5]. Large hemangiomas has been treated by supramaximal full-thickness wedge resection [6] or by reconstruction after resection using various techniques, including a mutual cross lip

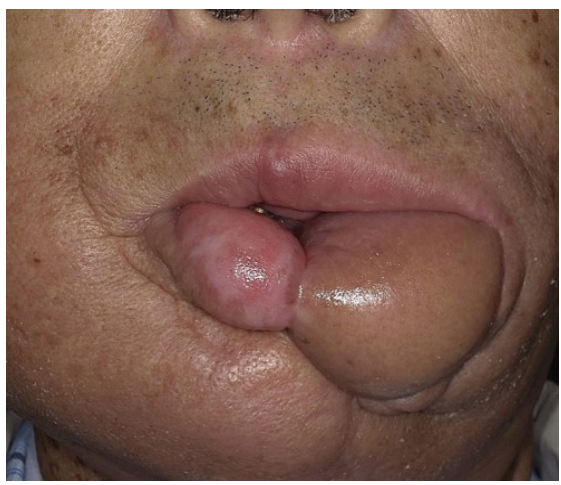

(a)

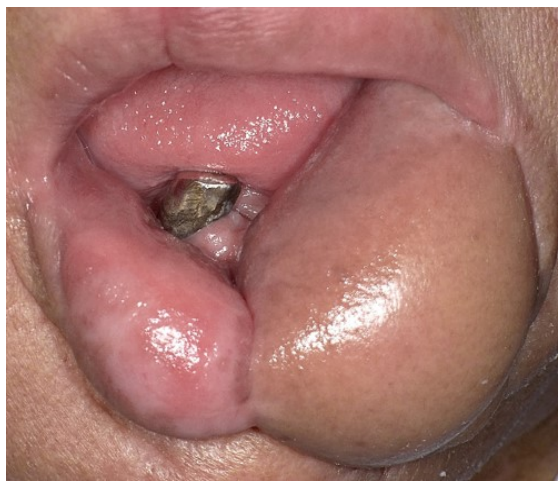

(b)

Figure 11. Lip finding at inital consultation in case 4. (a): Closing the mouth is difficult; (b): The flap was remarkable bulky and lower lip demonstrated eversion. 


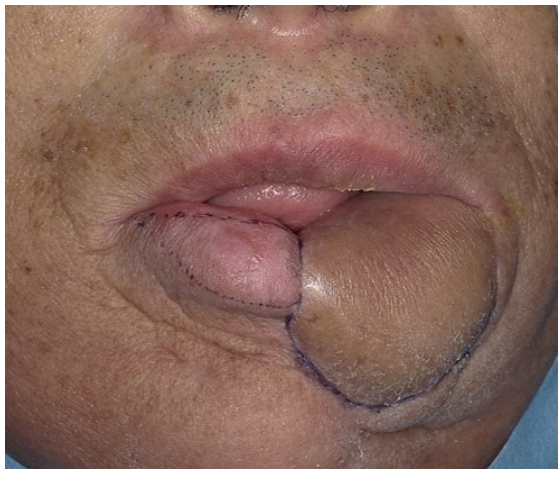

(a)

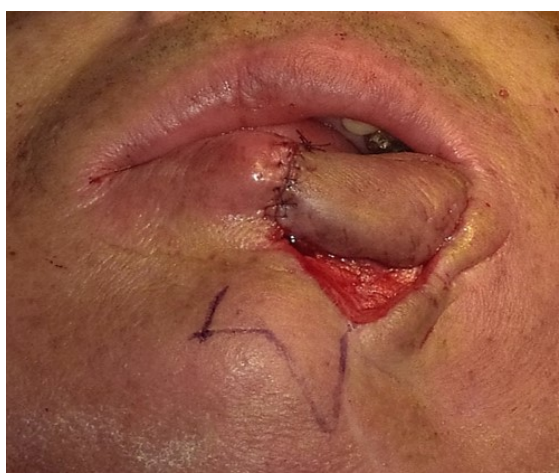

(c)

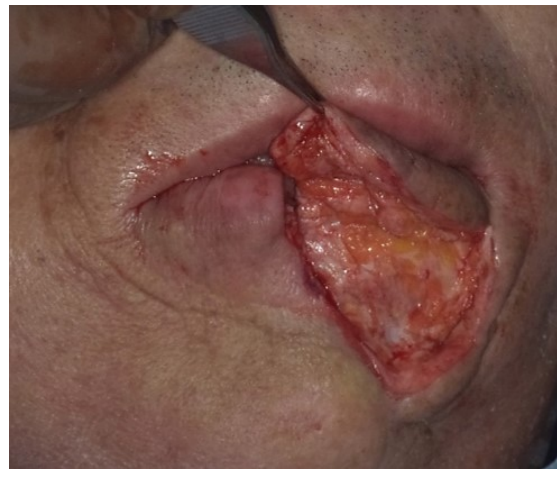

(b)

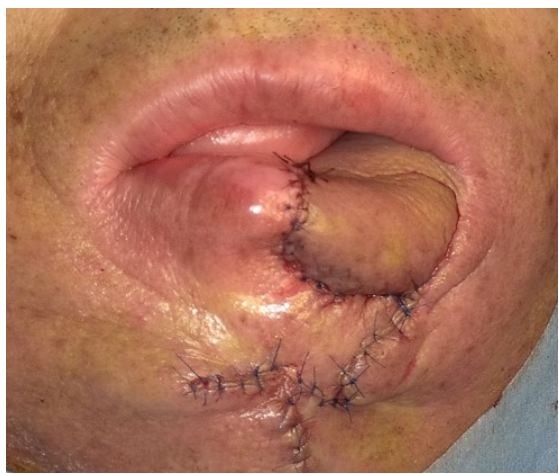

(d)

Figure 12. Operative findings in case 4. (a): Incision line; (b): Defatting in the flap; (c): Reduction of the flap and design of the local rotation flap from the local mental region; (d): After reduction of the flap volume, the raw surface is reconstructed with the local rotation flap.

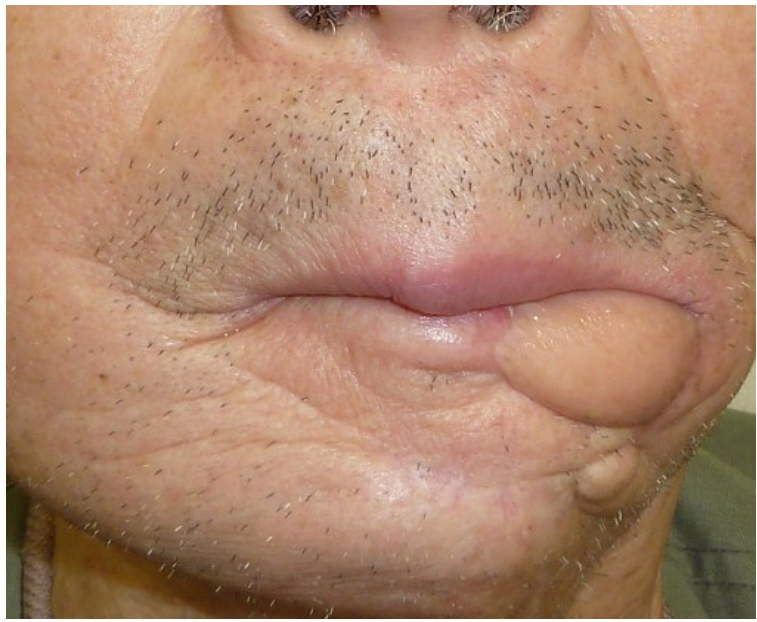

Figure 13. Postoperative findings in case 4 . Lip closure are satisfactory at 2 years postoperatively.

musculomucosal flap [7] or a forearm flap [8]. Our patient had a comparatively small tumor that limited to vermilion area of the lip. Therefore, we selected the wedge resection the borders of the tumor and performed labioplasty. As a result, the postoperative cosmetic and functional outcomes were satisfactory. 


\subsection{Posttraumatic Lip Deformity}

With regard to posttraumatic lip deformity, scarring and the loss of lip cause shortness of the lip [10]. It is necessary for satisfactory cosmetic outcomes to remove the scar and extend the amount of the lip. Our patient had deformity of the upper lip secondary to trauma. The lip findings were similar to those in patients with incomplete unilateral cleft lip, including elevation of the vermilion border toward the nasal base and narrowing of the white lip. Therefore, we selected Cronin's triangular-flap and a triangular skin flap [12] [13] [14] was designed $2 \mathrm{~mm}$ above the peak of Cupid's bow. The postoperative appearance of the upper lip was satisfactory. Thus, Cronin's method seems to be a useful technique for correcting posttraumatic upper lip deformity.

\subsection{Atrophic Lip Deformity}

Progressive hemifacial atrophy is a disease in which progressive degeneration affects the soft and hard tissues of the unilateral trigeminal nerve territory [16]. Our patient developed hemilateral atrophy of both lip due to progressive hemifacial atrophy. While hemilateral atrophy affected both lips, atrophy of the upper lip was especially severe. A cross-lip vermilion flap of the lower lip has been reported as a useful technique to correct the form in the presence of upper lip atrophy [2]. Therefore, we performed reconstruction of the upper lip to improve closure of the mouth, using Henry's cross-lip vermilion flap method [15]. Henry et al. [15] reported that sizeable defects can be easily filled a cross-lip vermilion flap, resulting in improved upper lip contour and simultaneously reduce the fullness of the lower lip to achieve better balance between two lips. In our patient, the postoperative appearance and function of the lips were satisfactory, with no deformity event on the affected side after surgery. To correct both appearance and function in patients with upper lip atrophy, raising a cross-lip vermilion flap from the non-atrophic lower lip seems to be a useful technique.

\subsection{Postoperative Lip Deformity}

Our patient showed postoperative deformity of the lower lip and difficulty closing mouth after resection of a tumor and reconstruction with a myocutaneous pectoralis major flap. The flap was extremely bulky. When reconstruction is done with a flap, it is often difficult to obtain the correct sharp of lip at initial procedure. Therefore, patients who have undergone lip reconstruction with a flap after tumor resection often require secondary correction. For postoperative lip deformity, some patients who underwent after oral cancer resection and reconstruction with flaps have been reported [11]. We performed debulking of the flap and the resulting raw surface was covered with a local rotation flap [11] [17] [18]. Postoperative lip closure was satisfactory, even though the flap remained slight bulky. We plane to perform further correction of the flap to improve the sharp of the lower lip.

In conclusion, these for cases suggest that it is important to perform appro- 
priate correction or reconstruction of lip deformities according to each patient's clinical features, in order to achieve good cosmetic and functional outcomes.

\section{Conclusion}

We managed four patients who had neoplastic, traumatic, atrophic and postoperative lip deformity, respectively. In patients with lip deformity, it is important to perform appropriate correction or reconstruction according to each patient's clinical features in order to achieve satisfactory cosmetic and functional outcomes.

\section{Conflict of Interest}

All authors report no conflict of interest related to this manuscript.

\section{References}

[1] Markus, A.F. and Delaire, J. (1993) Functional Primary Closure of Cleft Lip. The British Journal of Oral and Maxillofacial Surgery, 31, 281-291. https://doi.org/10.1016/0266-4356(93)90061-Z

[2] Jin, X., Teng, L., Lu, J., Xu, J., Zhang, C., Xu, M. and Zhao, J. (2013) Upper-Lip Vermilion Reconstruction with a Modified Cross-Lip Vermilion Flap in Hemifacial Atrophy. Journal of Plastic, Reconstructive and Aesthetic Surgery, 66, e101-e106.

[3] Teresa Min-Jung, O., Scheuermann-Pole, C., Tan, M. and Waner, M. (2013) Distribution, Clinical Characteristics, and Surgical Treatment of Lip Infantile Hemangiomas. JAMA Facial Plastic Surgery, 15, 292-304. https://doi.org/10.1001/jamafacial.2013.883

[4] Chang, C.S., Wong, A., Rohde, C.H., Ascherman, J.A., Wu, J.K. (2012) Management of Lip Hemangiamas: Minimizing Peri-Oral Scars. Journal of Plastic, Reconstructive and Aesthetic Surgery, 65, 163-168. https://doi.org/10.1016/j.bjps.2011.08.033

[5] Nakamura, N., Kawano, S., Nakao, Y., Tateishi, K. and Ohishi, M. (2005) An Alternative Method for Vermilion Reconstruction after Resection of Hemangiomas of Lip. Journal of Oral and Maxillofacial Surgery, 63, 1239-1243. https://doi.org/10.1016/j.joms.2005.04.017

[6] De Castro, D.K., Ng, Z.Y., Holzer, P.W., Waner, M., Cetrulo, C.L. and Fay, A. (2017) One-Stage Supramaximal Full-Thickness Wedge Resection of Vascular Lip Anomalies. Journal of Oral and Maxillofacial Surgery, 75, 2449-2455. https://doi.org/10.1016/j.joms.2017.03.020

[7] Ali, M., Mohammad, A.M., Maryam, M., Hamed, B. and Mahnaz, A. (2012) Repair of Large Lip Vermilion Defects with Mutual Cross Lip Musculomucosal Flap. World Journal of Plastic Surgery, 1, 3-10.

[8] Ueda, K., Oba, S., Ohtani, K., Amano, Y. and Fumiyama, Y. (2006) Functional Lower Lip Reconstruction with a Forearm Flap Combined with a Free Gracilis Muscle Transfer. Journal of Plastic, Reconstructive and Aesthetic Surgery, 59, 867-870. https://doi.org/10.1016/j.bjps.2005.10.014

[9] Kummoona, R. (2008) Posttraumatic Missile Injuries of Orofacial Region. The Journal of Craniofacial Surgery, 19, 300-305. https://doi.org/10.1097/SCS.0b013e3181577b97

[10] Stelnicki, E.J. and Zide, B. (1999) Reconstruction of the Posttraumatic Short Upper 
Lip. Annals of Plastic Surgery, 43, 592-597.

https://doi.org/10.1097/00000637-199912000-00003

[11] Fujiwara, T., Chen, C.C., Shih, H.S., Nebres, R.P., Jeng, S.F. and Kuo, Y.R. (2010) Stair-Step Flap for Secondary Lower Lip Revision after Lip and Cheek Composite Defects Reconstruction. Head \& Neck, 33, 1322-1327.

https://doi.org/10.1002/hed.21603

[12] Cronin, T.D. (1966) A Modification of Tennsion Type Lip Repairs. The Cleft Palate-Craniofacial Journal, 3, 376-382.

[13] Brauer, R.O. and Cronin, T.D. (1983) The Tennison Lip Repair Revisited. Plastic and Reconstructive Surgery, 71, 633-640. https://doi.org/10.1097/00006534-198305000-00009

[14] Matsunaga, K., Sasaguri, M., Mitsuyasu, T., Ohishi, M. and Nakamura, N. (2016) Upward Advancement of the Nasolabial Components at Unilateral Cleft Lip Repair Prevents Postoperative Long Lip. The Cleft Palate-Craniofacial Journal, 53, e71-e80. https://doi.org/10.1597/14-272

[15] Henry, K. and Kawamoto, Jr. (1979) Correction of Major Defects of the Vermilion with a Cross-Lip. Plastic and Reconstructive Surgery, 29, 5-13.

[16] Crikelair, G.F., Moss, M.L. and Khuri, A. (1962) Facail Hemiatrophy. Plastic and Reconstructive Surgery, 29, 5-13.

https://doi.org/10.1097/00006534-196201000-00002

[17] Colille, R.J. and Patel, R. (2011) The Mental Rotation Flap. Journal of Plastic, Reconstructive and Aesthetic Surgery, 64, e76-e77.

[18] Ransom, E.R. and Jancro, A.A. (2012) Double-Opposing Rotation-Advancement Flaps for Closure of Forehead Defects. Archives of Facial Plastic Surgery, 14, 342-345. https://doi.org/10.1001/archfacial.2012.7 